\title{
Cognitive Rehabilitation Based on Collaborative and Tangible Computer Games
}

\author{
Elena de la Guía \\ Computer Science Research Institute \\ University of Castilla-La Mancha \\ Albacete, Spain \\ mariaelena.guia@uclm.es
}

\author{
María Dolores Lozano, Victor R. Penichet \\ Computer Systems Department \\ University of Castilla-La Mancha \\ Albacete, Spain \\ maria.lozano@uclm.es, victor.penichet@uclm.es
}

\begin{abstract}
The world's population is getting older. As a consequence, certain diseases such as Alzheimer are getting more and more common. On the other hand, we can take advantage of the evolution of new technologies to develop applications with the aim of enhancing and stimulating the cognitive abilities of people suffering from Alzheimer's in order to counteract the progress of the disease. This paper presents an interactive system based on emerging technologies such as NFC and distributed user interfaces and tangible interaction to conduct cognitive rehabilitation. The main objective is to improve the person's performance in real-life situations and the associated enhancement in the person's well-being, self- esteem, mood and behavior.
\end{abstract}

Keywords- Alzheimer disease; Cognitive abilities; Tangible interaction; NFC technology; Distributed User Interfaces; Collaboration;

\section{INTRODUCTION}

The emerging demographic change towards an ageing population is introducing significant changes in our society. In recent years life expectancy has increased thanks to the great advances in medicine. The body's health has improved although it is not accompanied by an improvement in the health of the brain. Neurodegenerative diseases such as Alzheimer's disease (AD) are the most common form of dementia among older people. According to the World Health Organization [6], it is estimated that in $2005,0.379 \%$ of the world's population had dementia, and that the prevalence will increase to $0.441 \%$ in 2015 and to $0.556 \%$ in 2030 .

Alzheimer's disease is characterized by progressive loss of memory and other mental abilities; this loss results in atrophy of the affected regions. Nowadays, finding an effective therapy for Alzheimer's disease is a social need.

There are two types of therapies: pharmacological and nonpharmacological. The latter must be used in conjunction with the former. Non-pharmacological therapy is based on Neuropsychological rehabilitation (NR), which is concerned with the amelioration of the cognitive, emotional, psychosocial, and behavioral deficits caused by the deterioration of the brain.
The main reasons that explain this growing interest in cognitive rehabilitation are the limited efficacy of current drug therapies, the plasticity of the human central nervous system and the discovery that during ageing the connections in the brain are not fixed but retain instead the capacity to change with learning [5].

Information and Communication Technologies (ICT) can help elderly individuals to improve their quality of life, stay healthier, live independently for longer, and counteract the reduced capabilities which are more prevalent in ageing. ICT can enable them to remain active at work or in their community. Specifically the games are a way for users to perform tasks and learn in a funny, entertaining, and motivating way. The games can help the user in different ways:

-Improving the person's cognition, in areas such as memory, concentration, language skills and reasoning.

-Enhancing the person's ability to function in real-life everyday situations, helping to maintain the person's independence for as long as possible.

-Reducing distress and mood disturbances in the person with dementia, for instance, by reducing depression and anxiety, by increasing adjustment or coping in the early stages of dementia.

-Enhancing the person's quality of life and offering positive changes in the therapy, family, and so on.

\section{RELATED WORKS}

In literature we can find games that include training programs to improve cognitive abilities. Some examples are: Serious game [1], which focus on simulating real situations for people with limitations. S.M.A.R.T. Brain Games [4], a system to enhance cognitive abilities in children and adults that can be connected to the Playstation, X-Box or PC. Other type of games are based on virtual reality [3].

The advantages offered by these systems are numerous. They enhance positive attitudes in users while being appealing and encouraging, and providing information quickly. However the user needs a minimum knowledge about computer use. Not 
everybody can use a computer or some devices like the mouse or the keyboard, as they are not very intuitive for elderly people. They need someone to help them.

Due to the gap between elderly people and technology we have developed a Multi-Device Environment (MDE) based on cognitive stimulation games. The system is easy to use, intuitive and accessible thanks to new technologies such as NFC (Near Field Communication). Our proposal also includes the use of Tangible User Interfaces (TUI) within a Distributed User Interface (DUI) setting. TUI refers to user interfaces which give physical form to digital information, making the objects directly malleable and perceptible [2]. In the following sections, we present the system and its main features.

\section{CO-BRAIN TRAINING AND AZLGAME}

The system we present in this paper is composed of two interactive and collaborative games: Co-Brain Training Tool (Collaborative Brain Training Tool) and AlzGame tool are two kinds of interactive and collaborative games which compose a system. They are designed to support cognitive rehabilitation, specifically cognitive stimulation. This system is aimed at patients with Alzheimer and other dementias.

The system provides a new interaction technique that integrates distributed user interfaces from different devices, such as tangible interface that integrate NFC technology, mobile devices with NFC reader and a projector that displays the main game interface. The system is divided into two distinct games: The first is a the collaborative game called CoBrain Training tool, where multiple users can interact and play with other users and the system, it also offers the possibility to be used individually. The other one is the game called Alzgame where a user plays individually. The collaborative games also have a projector connected to a personal computer where the games are executed. Users interact with the system through the NFC tangible interface easily; it is only necessary to bring the mobile device closer to the corresponding image on the NFC interactive panel, and then the system recognizes it and displays the outcome of the game. The individual games are performed in a touch screen tablet. The user can interact through simple or multi-touch gestures by touching the screen with the fingers. This device is focused on more specific therapies.

\section{A. System requirements}

One of the main requirements to be considered for the development of such kind of application is simplicity and easiness. As elderly people lose their abilities, it is necessary to motivate them through amusing but simple user interfaces. Another important requirement is avoiding frustration because of failure. Appropriate applications should show positive and encouraging messages when the user fails. In this way, the user is motivated to continue playing and improving their cognitive abilities. Games should offer different levels of difficulty so that the users can exploit their potential effectively. Verbal messages are also used to facilitate the use of the game and get the attention, enthusiasm, and concentration of the user. Lastly, playing dynamics encourage Alzheimer's sufferers. These activities reinforce and enhance users' abilities through the games.

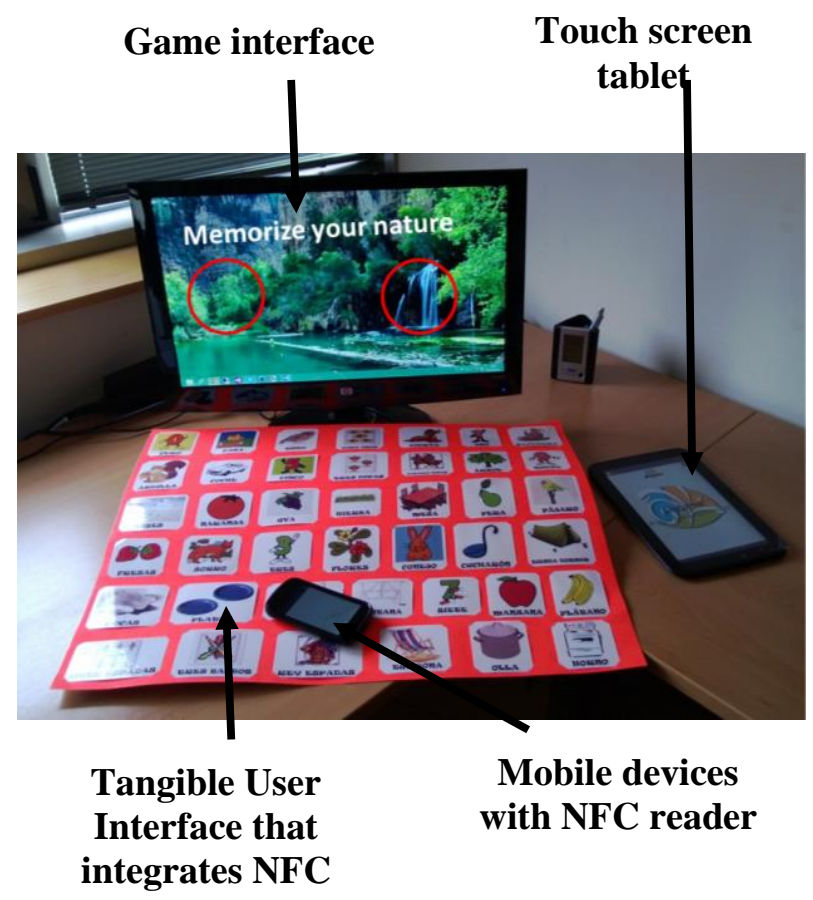

Figure 1. Collaborative games composed of the Game interface, Tangible User Interface based on NFC tags and Mobile devices with NFC reader incorporated and individual and specific games displayed on the touch screen tablet.

\section{B. Tangible Interaction}

The system allows two types of tangible interaction: one is based on tangible interfaces that integrate NFC technology and the other one uses tangible material.

- $\quad$ Tablet display interaction. AlzGame uses a tablet with touch screen. It is a device that allows both hands to interact directly with the display. This is focused on individual and specific therapies. It allows to monitor the game results and to configure different types of therapies depending on the patient. The user interacts with the device in an natural, easy and intuitive way. (See Figure 2)

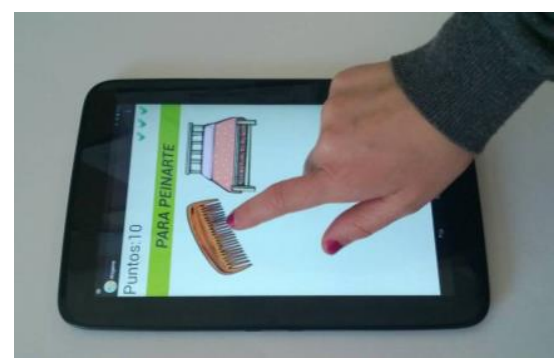

Figure 2: Touch screen tablet to play with the games easily 
- $\quad$ NFC tangible interfaces

These are common physical objects used as interaction resources to interact with the game. Each image contains an NFC tag inside (See Figure 3b) that indicates the function corresponding to the image. The interaction with the system is easy and intuitive as it is only necessary that the user brings the mobile device closer to the corresponding image in the tangible user interface.

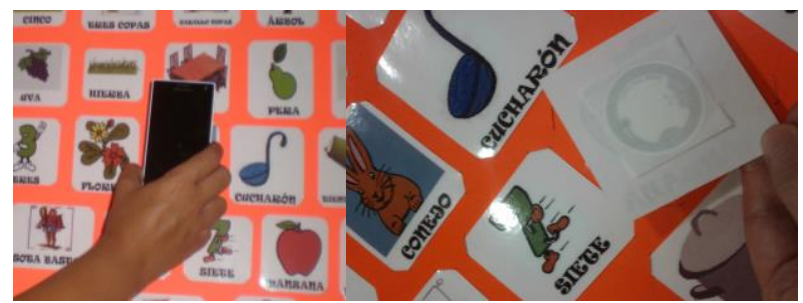

Figure 3: (a) Interaction Style. The user brings the mobile device closer to the tangible interface. (b) NFC tag integrated within each image of the Tangible interface.

\section{System architecture}

Co-Brain Training is a client-server system, designed as follows. The client system is the user mobile device with NFC reader. It is connected to the server application through a wireless network and communicates with the tangible interfaces via NFC when the user approaches the mobile device to the tangible interface. A tag is integrated inside each image of the tangible interface; each tag describes a function. When the NFC reader in the mobile device is brought closer to the chosen representative image, the NFC tag inside is excited by electromagnetic waves sent by the NFC reader and then, the mobile device executes the corresponding method in the server. It maps this information in the database and executes the steps necessary to return the information to the main game interface (See Figure 4).

AlzGame is also a client-server system. The touch screen tablet is based on Android technology. It executes the games and saves the results in the server. In this way, the therapist or caregiver can be constantly informed of the progress of the patient.

\section{Advantages}

The main advantages of using games as cognitive rehabilitation are described next:

- Patients improve the psychosocial factors, since collaborative games can interact with more users. In this way they are motivated and encouraged, so that the patient's mood improves.

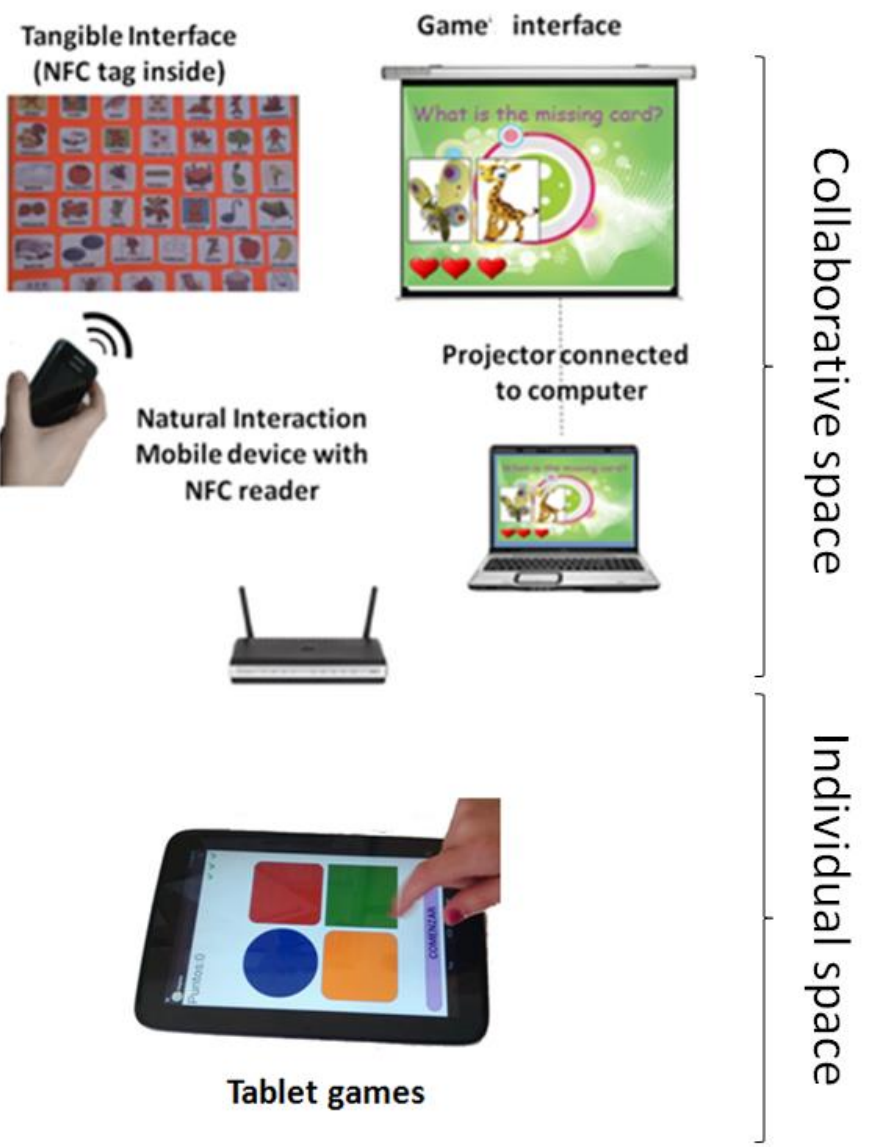

Figure 4: System architecture

- The new kind of interface reduces the cognitive load. This means that users have to rely more on the recognition skills than on their memory and they do not need to remember complicated abbreviations and codes. For this reason, the system has been designed in a very graphic way and using common objects which can be easily assimilated.

- Flexibility. It refers to the multiple ways in which the user and the system can exchange information. The information exchanged is displayed as text, voice, cheerful sounds or using graphics. The goal is the adaptation to any user, regardless of the disability or limitation he/she may have. The multiplayer feature of the game gives flexibility in the number of users. This allows to share and exchange experiences with other users. The situation of each user may be complex and variable and, for this reason, the game can also be used by just one player.

- Flexibility in terms of space. Players can be situated anywhere in the room, the only requirement is that the mobile device and the touch screen tablet are connected to the server. 
- Expandable. It offers the possibility to extend the games. The topic can be changed easily. The only requirement is that the NFC tag must be integrated in the tangible interfaces. In addition, NFC technology is very cheap to deploy.

- Interaction with the system is simple and intuitive. Common items are familiar and can be easily assimilated by users, making it more predictable to use. They do not need prior knowledge of the system or device.

- Cognitive stimulation when applied to the users can enhance mental abilities such as perception, attention, reasoning, abstraction, memory, language and orientation processes while optimizing their performance. This system is aimed at improving and training specific cognitive skills and its components, properly and consistently, can transform them into a skill and a habit.

\section{CONCLUSIONS}

Cognitive rehabilitation is a necessary activity for people with Alzheimer's disease. In order to provide users with appealing therapies, we have developed an intuitive and easyto- use system that is based on a set of computer games using a new interaction style.

The system is composed of two different tools. Co-Brain Training and AlzGame. The first tool is based on collaborative games. Its main objective is to improve the cognitive, emotional and psychosocial conditions of Alzheimer patients. It has been developed with NFC technology. The user only has to interact and bring the mobile device closer to tangible interfaces. The second tool 'AlzGame' consists of games executed on a touch screen tablet. This allows users to carry out more specific cognitive stimulation therapies. The new interaction style offered by the tools is simple and intuitive; its purpose is to eliminate the technological gap for elderly people and simultaneously improve the cognitive abilities of Alzheimer's patients.

\section{ACKNOWLEDGMENT}

This research has been partially supported by the Spanish CICYT research project TIN2011-27767-C02-01, from the Ministerio de Economía y Competitividad, and the regional projects with reference PPII10-0300-4174 and PII2C09-01851030, funded by the Junta de Comunidades de Castilla-La Mancha. We would also like to especially thank Francisco Vizcaino García and Cristina Sanchez.

\section{REFERENCES}

[1] Frederick Imbeault, Bruno Bouchard, Abdenour Bouzouane, Serious games in cognitive training for Alzheimer's patients, Proceedings of the 2011 IEEE 1st International Conference on Serious Games and Applications for Health, p.1-8, November 16-18, 2011 [doi>10.1109/SeGAH.2011.6165447]

[2] Ishii ,H. Ullmer ,B. Tangible bits: towards seamless interfaces between people, bits and atoms, Proceedings of the SIGCHI conference on Human factors in computing systems, p.234-241, March 22-27, 1997, Atlanta, Georgia, United States [doi>10.1145/258549.258715]

[3] Keshner EA. Virtual reality and physical rehabilitation: a new toy or a new research and rehabilitation tool?. J Neuroeng Rehabil. 2004;1:8-9

[4] S.M.A.R.T. Brain Games. http://www.cyberlearningtechnology.com, Feb 2004.

[5] Smith G. E., Housen P., Yaffe K., Ruff R., Kennison R. F., Mahncke H. W., Zelinski E. M. (2009).A cognitive training program based on principles of brain plasticity: results from the Improvement in Memory with Plasticity-based Adaptive Cognitive Training (IMPACT) study. J. Am. Geriatr. Soc. 57, 594-603. doi: 10.1111/j.15325415.2008.02081.x.

[6] World Health Organization (WHO). World report on disability; Enero2013. 